\title{
Caracterización Térmica De Vidrios Con Recubrimientos Metálicos
}

\author{
Enrique Hernández-Garfias, MSc \\ Carolina del Carmen Pérez-Sánchez, MBA \\ Temani Durán-Mendoza, MSc \\ Universidad Juárez Autónoma de México, \\ División Académica Multidisciplinaria de los Ríos \\ Juan Guzmán-Ceferino, PhD \\ Universidad Juárez Autónoma de México, \\ División Académica de Ciencias Agropecuarias \\ Manuel González-Pérez, PhD \\ Universidad Interserrana de Chichotla, México
}

Doi:10.19044/esj.2021.v17n21p305

Submitted: 26 April 2021

Accepted: 29 May 2021

Published: 30 June 2021
Copyright 2021 Author(s)

Under Creative Commons BY-NC-ND

4.0 OPEN ACCESS

Cite As:

Hernández-Garfias E., Carmen Pérez-Sánchez del C., Durán-Mendoza T., Guzmán-CeferinoJ. \& González-Pérez M. (2021). Caracterización Térmica De Vidrios Con Recubrimientos Metálicos. European Scientific Journal, ESJ, 17(21), 305.

https://doi.org/10.19044/esj.2021.v17n21p305

\section{Resumen}

Por la situación climática de las últimas décadas, las normas internacionales que regulan a los materiales de construcción son cada vez más estrictas. Actualmente, el vidrio es uno de los materiales de construcción más utilizados a nivel global y existen normas para evaluar su rendimiento térmico y así conocer su eficiencia para ahorrar energía. El Coeficiente de Transferencia de Calor (U) y el Coeficiente de Ganancia de Calor Solar (CGCS) son los principales parámetros térmicos que se utilizan para verificar si el rendimiento del material es satisfactorio en función de las normas vigentes y el mercado de vidrios. Para determinar estos parámetros se hicieron pruebas al exterior con un calorímetro solar diseñado y desarrollado para este fin. En este trabajo se evaluaron cinco muestras de vidrio: una muestra de vidrio claro (referencia) y cuatro muestras con recubrimientos de $\mathrm{ZnO}: \mathrm{Cu}$ y $\mathrm{ZnO}$ :Ag en distintas concentraciones y número de capas aplicadas, los cuales 
fueron desarrollados con el objetivo de reducir la ganancia de calor. Los resultados de la evaluación mostraron diferencias poco significativas entre la muestra de referencia y las muestras con recubrimientos. Por lo cual, se sugiere realizar modificaciones durante el desarrollo o la aplicación de los recubrimientos que permita mejorar las características de ganancia térmica.

Palabras-clave: Caracterización Térmica, Vidrio Con Recubrimiento, U, CGCS

\title{
Thermal Characterization Of Glasses With Metallic Coatings
}

\author{
Enrique Hernández-Garfias, MSc \\ Carolina del Carmen Pérez-Sánchez, MBA \\ Temani Durán-Mendoza, MSc \\ Universidad Juárez Autónoma de México,
}

División Académica Multidisciplinaria de los Ríos

Juan Guzmán-Ceferino, PhD

Universidad Juárez Autónoma de México,

División Académica de Ciencias Agropecuarias

Manuel González-Pérez, PhD

Universidad Interserrana de Chichotla, México

\begin{abstract}
Due to the climatic situation of the last decades, the international standards that regulate construction materials are increasingly strict. Currently, glass is one of the most used building materials in the world, and there are standards to evaluate its thermal performance and thus know its efficiency to save energy. The Thermal Transmittance (U-value) and the Solar Heat Gain Coefficient (SHGC) are the main thermal parameters used to verify if the performance of the material is satisfactory according to current standards and the glass market. To determine these parameters, outdoor tests were carried out with a solar calorimeter designed and developed for this purpose. In this work, five glass samples were evaluated: one clear glass sample (reference) and four samples with $\mathrm{ZnO}$ : $\mathrm{Cu}$ and $\mathrm{ZnO}$ : Ag coatings in different concentrations and number of applied layers, which were developed to reduce heat gain. The results of the evaluation showed not very significant differences between the reference sample and the samples with coatings. Therefore, it is suggested to carry out modifications during the development or application of the coatings to improve the thermal gain characteristics.
\end{abstract}

Keywords: Thermal Characterization, Glass With Coating, U-Value, SHGC 


\section{Introducción}

Desde hace siglos, el vidrio claro ha sido utilizado por la humanidad debido a las múltiples ventajas que presenta respecto a otros materiales, como lo son su apariencia estética, abundancia y costo de producción. Actualmente, el vidrio es uno de los materiales más utilizados en todo tipo de industria, especialmente en el sector de la construcción (Lollini et al., 2010). Sin embargo, debido al deterioro ambiental a nivel global, las normas nacionales e internacionales que rigen el uso de los materiales de construcción se han vuelto más severas. Por esta razón, la tendencia mundial en el sector de la construcción está orientada cada vez más hacia el uso de materiales que permitan el ahorro de energía, área en donde el vidrio claro puede presentar características negativas debido a sus propiedades térmicas y ópticas, por lo que resulta importante optimizar el rendimiento de los sistemas vidriados (Grynning et al., 2013). Debido a esto, desde hace varias décadas se han desarrollado proyectos de investigación en todo el mundo, con el objetivo de mitigar los efectos de las propiedades térmicas y ópticas del vidrio claro, en donde principalmente se considera el Coeficiente Global de Transferencia de Calor $(U)$ y el Coeficiente de Ganancia de Calor Solar (CGCS), respectivamente (Tait, 2006; Lollini et al., 2010).

Para medir los parámetros característicos del vidrio, principalmente existen dos métodos: la calorimetría al interior y la calorimetría al exterior. La calorimetría al interior se encarga de simular las condiciones a las que se expone la muestra, pero esta simulación puede ser imprecisa ya que no es posible simular completamente el comportamiento de la irradiancia solar y todos los parámetros adicionales del entorno. Por otra parte, la calorimetría al exterior se puede realizar con dos instrumentos, con una celda PASLINK o con un calorímetro solar. La celda PASLINK es una habitación de tamaño real que utiliza sensores de temperatura para medir la transferencia de calor de áreas representativas, sin embargo, su masividad térmica ocasiona que el análisis de las muestras pueda durar varios días. El calorímetro solar es un dispositivo que puede ser de menor tamaño comparado con una celda PASLINK, y suele construirse considerando el tamaño de las muestras que se analizarán. Debido a su baja masa térmica, el sistema puede estabilizarse en menor tiempo, por lo que, es posible obtener los parámetros térmicos en un solo día, sin comprometer la precisión de los resultados (Hernández-Garfias et al., 2019).

Con el fin de modificar los valores del $U$ y el CGCS, se han desarrollado múltiples sistemas, como los sistemas de sombreado, los vidrios dobles o triples y los vidrios con recubrimiento. Implementar sistemas de sombreado, así como vidrios dobles o triples, puede ser muy costoso por la complejidad del sistema, por los materiales de construcción o por la dificultad que puede representar la adquisición e instalación. Por otra parte, los vidrios 
con recubrimiento pueden ser más accesibles dependiendo del material utilizado y no requieren conocimiento especializado para su instalación. Debido a esto, pueden ser una opción adecuada para reducir las ganancias de calor y, de esta manera, ahorrar energía al disminuir la carga de refrigeración requerida en una edificación. Por lo anterior, es de interés evaluar térmicamente estos vidrios para obtener sus parámetros térmicos y poder compararlos en funcionalidad con los vidrios comerciales.

En el Centro de Investigación de Ciencia y Tecnología Aplicada de Tabasco (CICTAT), México, se desarrollaron cuatro muestras de vidrio con recubrimientos metálicos, a partir de las cuales se buscan reducir las ganancias de calor hacia el interior de las edificaciones. Por lo que, en este trabajo se realizó la evaluación térmica de las cuatro muestras de vidrios con recubrimientos metálicos para conocer los valores de los parámetros $U$ y CGCS. Para ello, se utilizó un calorímetro solar desarrollado por HernándezGarfias et al. (2019).

\section{Materiales y Métodos \\ Muestras}

Las muestras utilizadas fueron cinco vidrios de $15 \times 15 \mathrm{~cm}$ y $3 \mathrm{~mm}$ de espesor, una de ellas fue la muestra de referencia (vidrio claro) y el resto fueron muestras de vidrio con recubrimientos metálicos, las cuales fueron desarrolladas en CICTAT, México. En la Figura 1 se muestra la fotografía de cada una de las muestras. Los recubrimientos se depositaron utilizando el método de aspersión, sus características pueden verse en la Tabla 1.

\begin{tabular}{|c|c|c|c|c|}
\hline $\begin{array}{c}\text { Vidrio claro } \\
\text { a) }\end{array}$ & $\begin{array}{c}\text { Cu I } \\
\text { b) }\end{array}$ & $\begin{array}{c}\text { Cu III } \\
\text { c) }\end{array}$ & $\begin{array}{c}\text { Ag I } \\
\text { d) }\end{array}$ & $\begin{array}{c}\text { Ag III } \\
\text { d) }\end{array}$ \\
\hline & & & & \\
& & & & \\
& & & & \\
\hline
\end{tabular}

Figura 1. Muestras de vidrio utilizadas: a) muestra de referencia, b)-d) muestras con recubrimientos metálicos

Tabla 1. Características de las muestras de vidrio utilizadas.

\begin{tabular}{|c|c|c|c|c|c|}
\hline $\begin{array}{c}\text { Nombre } \\
\text { de la } \\
\text { muestra }\end{array}$ & Recubrimiento & $\begin{array}{c}\text { Composición } \\
\text { del } \\
\text { recubrimiento }\end{array}$ & $\begin{array}{c}\text { Proporción } \\
\text { de } \\
\text { composición }\end{array}$ & $\begin{array}{c}\text { Concentración } \\
\left.\mathbf{( m L}^{-1}\right)\end{array}$ & $\begin{array}{c}\text { Número } \\
\text { de capas }\end{array}$ \\
\hline $\begin{array}{c}\text { Vidrio } \\
\text { claro }\end{array}$ & No & - & - & - & - \\
\hline $\mathrm{Cu} \mathrm{I}$ & Sí & Zinc y Cobre & $\begin{array}{c}61.34 \% \mathrm{ZnO} \\
38.66 \% \mathrm{Cu}\end{array}$ & 0.180 & 1 \\
\hline $\mathrm{Cu}$ III & Sí & Zinc y Cobre & $61.34 \% \mathrm{ZnO}$ & 0.180 & 3 \\
\hline
\end{tabular}




\begin{tabular}{|c|c|c|c|c|c|}
\hline & & & $38.66 \% \mathrm{Cu}$ & & \\
\hline Ag I & Sí & Zinc y Plata & $\begin{array}{c}60 \% \mathrm{ZnO} \\
40 \% \mathrm{Ag}\end{array}$ & 0.176 & 1 \\
& Sí & Zinc y Plata & $\begin{array}{c}60 \% \mathrm{ZnO} \\
40 \% \mathrm{Ag}\end{array}$ & 0.176 & 3 \\
\hline
\end{tabular}

\section{Calorímetro solar}

El calorímetro solar utilizado fue el desarrollado por HernándezGarfias et al. (2019), para el cual se consideró la norma NFRC-201-2014 para su construcción e instrumentación. En la Figura 2 se muestra el calorímetro solar y sus componentes.

\section{Determinación del $U$ y del CGCS}

El Coeficiente Global de Transferencia de Calor $(U)$ es un parámetro térmico que puede definirse como el inverso de la resistencia térmica total de un elemento,

$$
U=\frac{1}{R_{T}}
$$

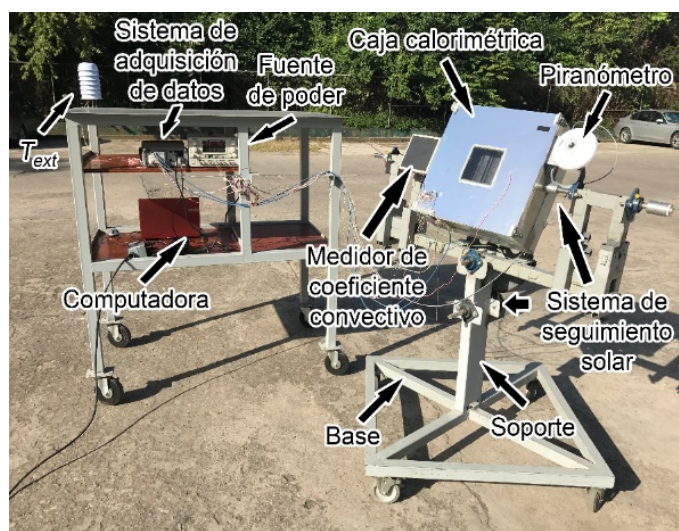

Figura 2. Calorímetro solar utilizado en este proyecto (Hernández-Garfias et al., 2019)

Para determinar el $U$ se realizaron dos pruebas distintas al interior, utilizando una resistencia eléctrica para generar una diferencia de potencial que provocase una diferencia de flujo de calor $\left(q_{\text {ext }}-q_{\text {int }}\right)$. De acuerdo con (Standardization, n.d.), se utilizó la Ecuación (2) para determinar el $U$.

$$
U=\frac{q_{\text {ext }}-q_{\text {int }}}{\left(\Delta T_{\text {int }- \text { ext }}\right)\left(A_{M}\right)}
$$

donde $A_{M}$ es el área de la muestra y $q_{\text {ext }}-q_{\text {int }}$ son el flujo de calor que sale y el flujo de calor que entra a la caja calorimétrica (CC), respectivamente. 
El Coeficiente de Ganancia de Calor Solar (CGCS) es un parámetro derivado de la radiación solar incidente (irradiancia). El CGCS representa la razón entre la irradiancia en un material y la radiación que logra atravesarlo, su valor puede ir desde 0 hasta 1 . Además, este parámetro involucra parámetros ópticos como lo son la absortancia, la transmitancia y la reflectancia. Por otra parte, el cálculo experimental puede realizarse considerando parámetros térmicos de acuerdo con (Macias-Melo y FloresPrieto, 2013).

$$
C G C S=\frac{q_{M}-A_{M} \cdot U_{M}\left(\Delta T_{\text {ext-int }}\right)}{A_{M} \cdot I_{S}}
$$

donde $q_{M}$ representa el flujo de calor que atraviesa el objeto de estudio, $U_{M}$ es el coeficiente global de transferencia de calor del objeto de estudio, $I_{S}$ es la irradiancia solar y $\Delta T_{\text {int-ext }}$ es la diferencia de temperatura entre la temperatura del ambiente y la temperatura del interior de la caja calorimetrica (CC).

Las pruebas experimentales para determinar el CGCS se desarrollaron en días soleados con cielo despejado (sin nubosidad). La muestra fue alineada con respecto al sol para recibir una irradiancia solar normal con respecto a su superficie. El ángulo cenital y azimutal fueron ajustados cada 10 minutos en el transcurso del experimento para garantizar la máxima captación de irradiancia solar. Durante las mediciones se utilizó un ventilador tangencial en el interior de la CC para conseguir un flujo de aire homogéneo. Posteriormente, se inició la adquisición y monitoreo de las temperaturas y de la Fuerza Electromotriz (FEM) de los transductores de flujo de calor. La prueba finalizó cuando la irradiancia solar se encontró por debajo de $800 \mathrm{Wm}^{-}$ 2 .

\section{Resultados y Discusión Vidrio claro}

Para la determinación del $U$, en las Figuras 3 y 4 se muestra la diferencia de temperatura ( $T_{\text {ext }}-T_{\text {int }}$ ) y el flujo de calor (q) que se presentó a través de la muestra $\left(\mathrm{P}_{\mathrm{M}}\right)$, obtenidos en la Prueba 1-U y 2-U, respectivamente. La Prueba 1-U se realizó suministrando $10 \mathrm{~W}$ a la resistencia, mientras que en la Prueba 2-U se suministraron $20 \mathrm{~W}$. El comportamiento de $T_{\text {ext }}-T_{\text {int }}$ presentó diferencias significativas entre ambas pruebas, siendo el máximo de $8.28^{\circ} \mathrm{C}$ y $13.71^{\circ} \mathrm{C}$ para las pruebas $1-\mathrm{U}$ y $2-\mathrm{U}$, respectivamente. Estas diferencias se atribuyen principalmente a la energía suministrada al sistema mediante la resistencia eléctrica para amplificar la diferencia de flujo de calor. Por otra parte, el flujo de calor a través de la muestra se comportó de manera similar a la temperatura, aumentando de acuerdo con la potencia suministrada, obteniendo un máximo de $1.27 \mathrm{~W}$ y $2.29 \mathrm{~W}$ para las pruebas 1- 
U y 2-U, respectivamente. Con los valores obtenidos de flujo de calor y diferencia de temperatura, se obtuvieron los valores del $U$ mediante (2). El valor del $U$ en las pruebas 1 -U y 2-U fue de $6.86 \mathrm{Wm}^{-2}{ }^{\circ} \mathrm{C}^{-1}$ y $7.05 \mathrm{Wm}^{-2}{ }^{\circ} \mathrm{C}^{-1}$, respectivamente. Estos valores se encuentran dentro del intervalo reportado por otros autores (Marinoski et al., 2012; Macias-Melo y Flores-Prieto, 2013) y el software WINDOW (Lawrence Berkeley National Laboratory, 2018).

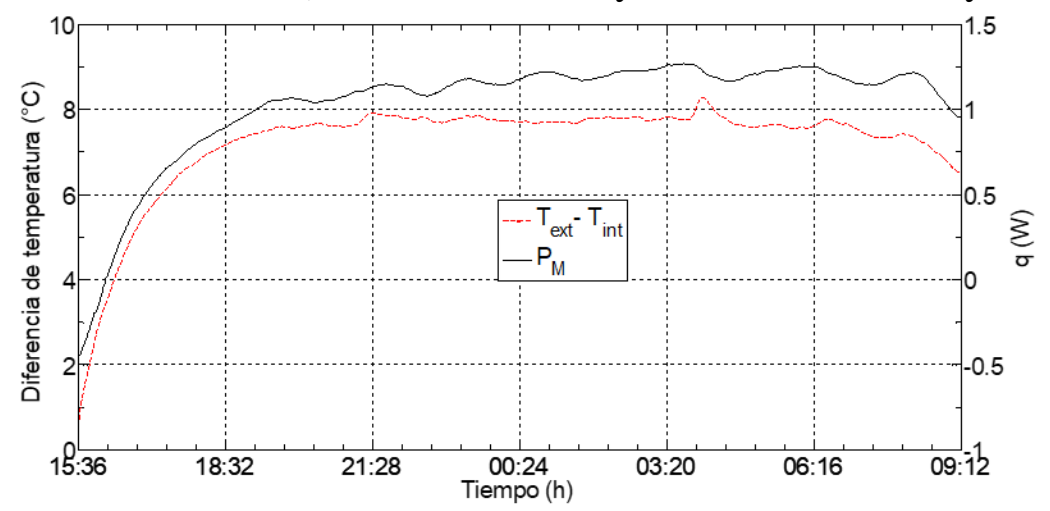

Figura 3. Diferencia de temperatura y flujo de calor total durante la Prueba 1-U

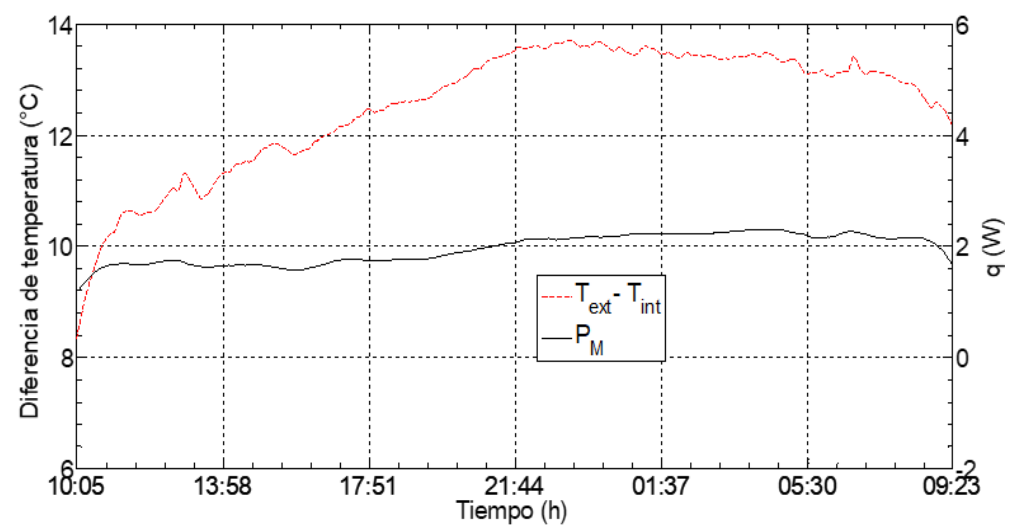

Figura 4. Diferencia de temperatura y flujo de calor total durante la Prueba 2-U

A continuación, se muestran los resultados de las pruebas experimentales al exterior utilizadas para determinar el CGCS de la muestra de vidrio claro. Los resultados de las Pruebas 1-S y 2-S se dividieron en dos gráficas distintas, en la Figura 5 se muestra el comportamiento de la irradiancia solar y la temperatura ambiente durante la Prueba 1-S para el día 18 de agosto del 2017. El comportamiento presentado corresponde al periodo de mayor estabilidad durante la prueba. Como se puede observar, la irradiancia solar promedio obtenida fue de $966 \mathrm{Wm}^{-2}$. Mientras que la temperatura ambiente osciló entre $33.6^{\circ} \mathrm{C}$ y $34.8^{\circ} \mathrm{C}$. Por otra parte, en la Figura 6 se aprecia el comportamiento del flujo de calor total que atraviesa la muestra. Se puede observar que este flujo de calor presentó un promedio de $16.97 \mathrm{~W}$. 


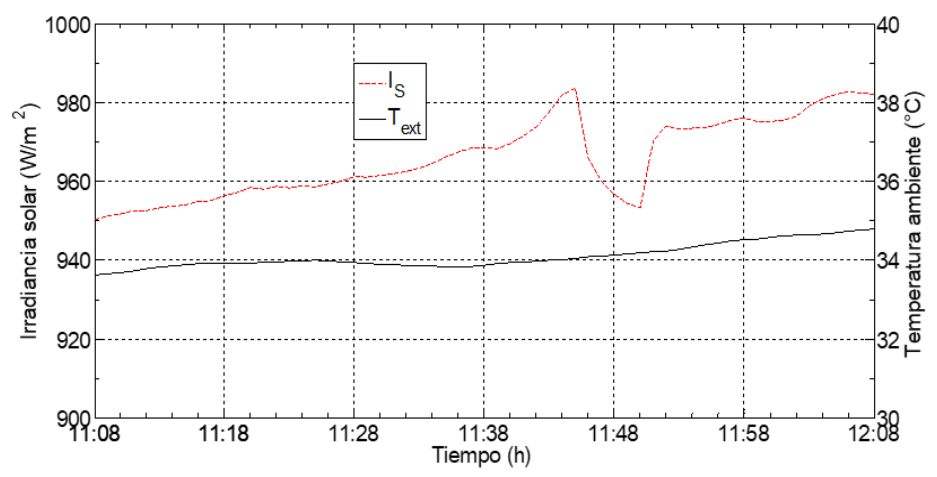

Figura 5. Irradiancia solar y temperatura ambiente durante la Prueba 1-S

Del mismo modo, la Figura 7 y 8 muestran los resultados obtenidos en la Prueba 2-S del día 24 de agosto del 2017, para las condiciones ambientales y el flujo de calor total, respectivamente. Como se puede observar, la irradiancia solar promedio fue de $979 \mathrm{Wm}^{-2}$, mientras que la temperatura ambiente osciló entre $33.7^{\circ} \mathrm{C}$ y $34.7^{\circ} \mathrm{C}$. Por otra parte, el promedio del flujo de calor total obtenido fue de $17.21 \mathrm{~W}$. Se puede observar que la diferencia de la irradiancia solar promedio y la diferencia del flujo de calor total obtenidos en ambas pruebas fueron de $13 \mathrm{Wm}^{-2}$ y $0.24 \mathrm{~W}$ mayores en la Prueba 2-S, sin embargo, las diferencias fueron poco significativas.

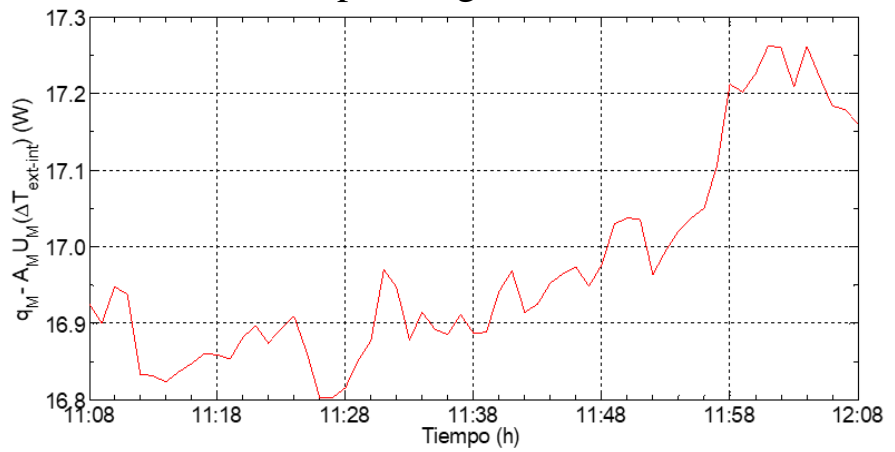

Figura 6. Flujo de calor total que atraviesa la muestra durante la Prueba 1-S

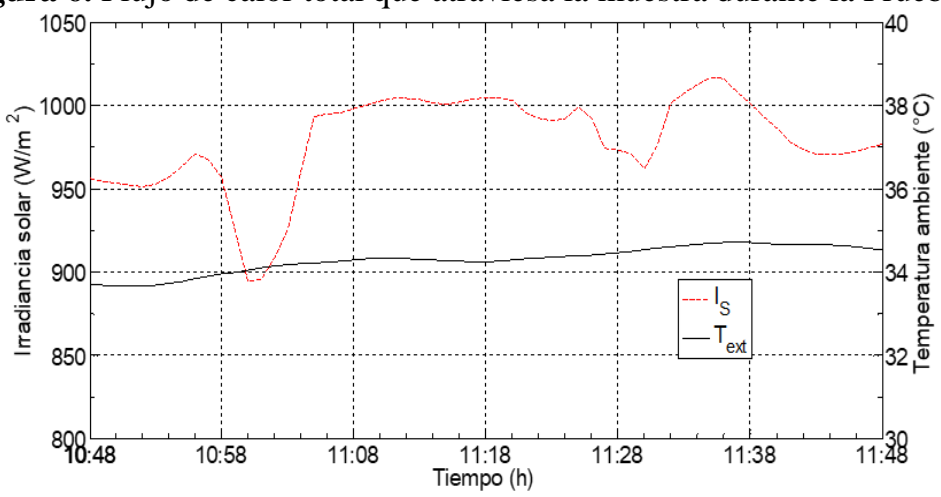

Figura 7. Irradiancia solar y temperatura ambiente durante la Prueba 2-S 


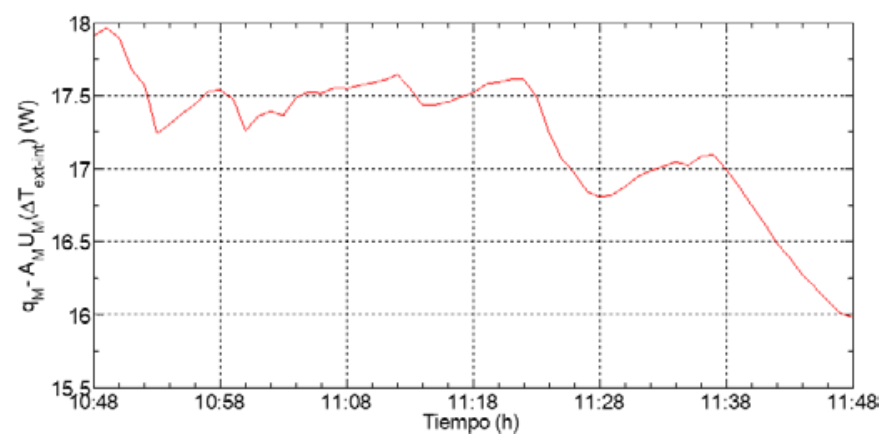

Figura 8. Flujo de calor total que atraviesa la muestra durante la Prueba 2-S

Se determinó el CGCS con (3), utilizando un valor promedio de $U$ de $6.95 \mathrm{Wm}^{-2}{ }^{\circ} \mathrm{C}^{-1}$. Los valores de CGCS promedio obtenidos para la Prueba 1-S y 2-S fueron 0.799 y 0.806 , respectivamente, con un valor promedio entre ellos de 0.803 . Se puede observar que la diferencia obtenida del CGCS entre ambas pruebas fue de $0.87 \%$, por lo que no presentan diferencias significativas.

Por otra parte, cabe destacar que los CGCS obtenidos son similares a los valores reportados en trabajos experimentales similares. Marinoski et al. (2012) reportó un CGCS promedio de 0.826 para una muestra de $1.8 \mathrm{~m}^{2}$ de área superficial, por lo que la diferencia obtenida entre sus resultados y los de este estudio fue de $2.78 \%$. Adicionalmente, se realizó una comparación del CGCS promedio obtenido en este estudio con el reportado en la base de datos de WINDOW, el cual es un software para calcular los índices de rendimiento térmico de ventanas. WINDOW reporta un CGCS de 0.87 para un vidrio claro marca Vitro ${ }^{\circledR}$ de $3 \mathrm{~mm}$, por lo que existe una diferencia de $7.7 \%$. Esta diferencia se atribuye a que en el estudio experimental se presentan múltiples reflexiones debido a las propiedades ópticas del vidrio y de la instrumentación interna del calorímetro debido a que no toda la energía que atraviesa la muestra inicialmente se mantiene en el interior para ser cuantificada.

\section{Vidrios con recubrimientos metálicos}

Las pruebas experimentales para las muestras con recubrimientos metálicos fueron realizadas siguiendo el mismo procedimiento que para la muestra de vidrio claro. En la Tabla 2 se presentan los resultados obtenidos de los parámetros característicos de las muestras estudiadas.

Tabla 2. Parámetros característicos de las muestras estudiadas en este proyecto

\begin{tabular}{|c|c|c|c|c|}
\hline $\begin{array}{c}\text { Nombre de } \\
\text { la muestra }\end{array}$ & $\begin{array}{c}\text { No. de capas de } \\
\text { recubrimiento }\end{array}$ & $\begin{array}{c}\text { Concentración } \\
\left(\mathbf{m L}^{-\mathbf{1}}\right)\end{array}$ & $\begin{array}{c}\mathbf{U} \\
\left(\mathbf{W m}^{-\mathbf{2}}{ }^{\circ} \mathbf{C}^{\mathbf{- 1}}\right)\end{array}$ & $\mathbf{C G C S}$ \\
\hline Vidrio claro & - & - & 6.95 & 0.803 \\
\hline $\mathrm{Cu}$ I & 1 & 0.180 & 6.81 & 0.805 \\
\hline $\mathrm{Cu}$ III & 3 & 0.180 & 6.79 & 0.804 \\
\hline $\mathrm{Ag} \mathrm{I}$ & 1 & 0.176 & 6.82 & 0.800 \\
\hline Ag III & 3 & 0.176 & 6.81 & 0.801 \\
\hline
\end{tabular}


Los resultados del $U$ y CGCS obtenidos para las muestras con recubrimientos metálicos presentaron diferencias poco significativas entre sí, incluso en comparación con la muestra de vidrio claro. Estos resultados indican que el número de capas de recubrimiento aplicadas en los sustratos de vidrio prácticamente no modifica las propiedades térmicas de las muestras. De acuerdo con esto, se sugiere realizar modificaciones durante el desarrollo o la aplicación del recubrimiento, específicamente en la concentración de las soluciones o en el número de capas. Lo anterior, con el fin de mejorar el comportamiento térmico del vidrio con recubrimiento, lo cual permita evidenciar una mejora en su comportamiento térmico respecto al vidrio convencional (vidrio claro).

\section{Conclusiones}

En este trabajo se realizó la caracterización térmica de muestras de vidrio con recubrimientos metálicos, utilizando un calorímetro solar, con lo cual fue posible analizar su comportamiento. Para ello, se determinó el $U$ y el CGCS de una muestra de referencia (vidrio claro de $3 \mathrm{~mm}$ ) con el fin de identificar si los vidrios con recubrimientos metálicos presentaban mejores propiedades térmicas para el ahorro de energía. Los valores promedio obtenidos del $U$ y del CGCS para el vidrio claro de $3 \mathrm{~mm}$ fueron de $6.95 \mathrm{Wm}^{-}$ $2{ }^{\circ} \mathrm{C}^{-1}$ y 0.803 , respectivamente. Estos valores obtenidos fueron similares con los valores reportados en trabajos experimentales y con los valores reportados en el software WINDOW para una muestra de vidrio claro de $3 \mathrm{~mm}$. La calibración y determinación de los parámetros característicos de esta muestra de referencia permitió verificar el adecuado funcionamiento del calorímetro solar, lo que a su vez permitió utilizarlo para la caracterización térmica de las muestras de vidrios con recubrimientos metálicos. Por su parte, cuando se determinaron los parámetros característicos de las muestras de vidrio con recubrimientos metálicos (Cu I, Cu III, Ag I y Ag III), se encontraron diferencias poco significativas entre ellas, así como con respecto a los valores obtenidos de la muestra de vidrio de referencia. Por lo anterior, se concluye que las propiedades térmicas de las muestras de vidrio con recubrimientos metálicos no presentan una diferencia significativa respecto a la muestra de vidrio claro, por lo tanto, tampoco presentan una ventaja dentro del mercado de vidriado que justifique un precio mayor al del vidrio claro. Los parámetros característicos podrían mejorarse si se realizan modificaciones durante el desarrollo de cada tipo de recubrimiento, específicamente en la concentración de las soluciones o en el número de capas. 


\section{References:}

1. Grynning, S., Gustavsen, A., Time, B., y Jelle, B. P. (2013). Windows in the buildings of tomorrow: Energy losers or energy gainers? Energy and Buildings, 61, 185-192.

a. https://doi.org/10.1016/j.enbuild.2013.02.029

2. Hernández-Garfias, E., Macias-Melo, E. V., Aguilar-Castro, K. M., Hernández-Pérez, I., Serrano-Arellano, J., y Diaz-Flores, L. L. (2019). Development of a solar calorimeter for the thermal evaluation of glazing samples. Journal of Building Physics, 42(6), 750-770.

a. https://doi.org/10.1177/1744259118823812

3. Lawrence Berkeley National Laboratory. (2018). WINDOW Windows and Daylighting.

4. Lollini, R., Danza, L., y Meroni, I. (2010). Energy efficiency of a dynamic glazing system. Solar Energy, 84(4), 526-537.

a. https://doi.org/10.1016/j.solener.2009.12.006

5. Macias-Melo, E. V., y Flores-Prieto, J. J. (2013). Solar Calorimeter for Thermal Testing of Glazings. Journal of Enhanced Heat Transfer, 20(6), 499-509. https://doi.org/10.1615/JEnhHeatTransf.2015007462

6. Marinoski, D. L., Güths, S., y Lamberts, R. (2012). Development of a calorimeter for determination of the solar factor of architectural glass and fenestrations. Building and Environment, 47(1), 232-242.

a. https://doi.org/10.1016/j.buildenv.2011.07.017

7. Standardization, I. O. for. (n.d.). ISO 10292-1994 (E): Glass in building - Calculation of steady-state U-values (thermal transmittance) of multiple.

8. Tait, D. B. (2006). Solar heat gain coefficient measurements for glazings with indoor window attachment products. ASHRAE Transactions, 112 PART 2, 116-121. 